\title{
Institucionalización del
} aprendizaje servicio en la Facultad de Ciencias Empresariales

\section{de la Universidad del Bío-Bío: vinculando la educación superior y la comunidad local ${ }^{*}$}

\author{
CARLOS MAURICIO SALAZAR-BOTELLO** \\ YOHANA ANDREA MUÑOZ JARA*** \\ MARÍA TERESA LAGOS TRONCOSO**** \\ RAÚL LUCIANO ARRIAGADA INOSTROZA***** \\ ROSANA LORENA VALLEJOS CARTES****** \\ RODRIGO JAVIER MONJE-SANHUEZA*******
}

* Este artículo de reflexión deriva del Plan de Mejoramiento UBB 1402 "Desarrollo de competencias de los estudiantes de la Facultad de Ciencias Empresariales (FACE) a través de la metodología Aprendizaje Servicio (As) y el uso de tecnologías de la información”, financiado por el Banco Mundial a través del Programa Mecesup del Ministerio de Educación de Chile.

** Magíster en Desarrollo y Comportamiento Organizacional. Académico de la Facultad de Ciencias Empresariales de la Universidad del Bío-Bío, Chile.

Correo electrónico: mauricio@ubiobio.cl ORCID: https://orcid.org/0000-0002-5659-7331

*** Magíster en Gestión de Empresas. Profesional de la Facultad de Ciencias Empresariales de la Universidad del Bío-Bío, Chile.

Correo electrónico:ymunoz@ubiobio.cl ORCID: https://orcid.org/0000-0003-3897-8840

**** Magíster en Gestión Educacional. Profesional de la Facultad de Ciencias Empresariales de la Universidad del Bío-Bío, Chile.

Correo electrónico: mtlagos@ubiobio.cl

ORCID: https://orcid.org/0000-0003-2487-7395 
Artículo de reflexión sobre la implementación de la metodología Aprendizaje Servicio en la Educación Superior.

Recibido: 1 de mayo de 2020 - Evaluado: 10 de julio de 2020 - Aceptado: 24 de julio de 2020

Citar como: Salazar-Botello, C., Muñoz, Y., Lagos, M., Arriagada, R., Vallejos, R. y Monje-Sanhueza, R. (2021). Institucionalización del aprendizaje servicio en la Facultad de Ciencias Empresariales de la Universidad del Bío-Bío: vinculando la educación superior y la comunidad local. Hallazgos, 18(35), 287-318. https://doi.org/10.15332/2422409X.5770

***** Magíster en Educación, con mención en Gestión Curricular. Profesional de la Facultad de Ciencias Empresariales de la Universidad del Bío-Bío, Chile.

Correo electrónico: raularriagada@ubiobio.cl

ORCID: https://orcid.org/0000-0003-2409-8340

****** Magíster en Planificación y Desarrollo Rural. Docente de la Facultad de Ciencias Empresariales de la Universidad del Bío-Bío, Chile.

Correo electrónico: rvallejos@ubiobio.cl ORCID: https://orcid.org/0000-0002-4821-314X

******* Magíster en Dirección de Empresas. Profesional de la Universidad del Bío-Bío, Chile.

Correo electrónico: rmonje@ubiobio.cl

ORCID: https://orcid.org/0000-0003-0868-9386 
Institucionalización del aprendizaje servicio en la Facultad de Ciencias Empresariales...

\section{Resumen}

La metodología aprendizaje servicio ofrece innumerables oportunidades a la educación superior para reforzar el proceso enseñanza aprendizaje mediante el vínculo entre el estudiantado y la comunidad. Permite ampliar los ámbitos de referencia del trabajo docente, pasando desde la sala de clases hasta el trabajo en terreno, lo que lleva a reconocer las particularidades del entorno y descubrir las necesidades de la comunidad local. El propósito de este trabajo es describir las acciones desarrolladas por la Facultad de Ciencias Empresariales de la Universidad del Bío-Bío de Chile para la incorporación e institucionalización de la metodología aprendizaje servicio como parte del desarrollo de sus asignaturas. Los resultados indican que a la fecha han participado 901 estudiantes y 19 docentes, quienes trabajan colaborativamente con socios comunitarios en jornadas de capacitación y asesorías específicas. A la fecha se han atendido 276 socios comunitarios pertenecientes a 20 instituciones de las regiones del Biobío y de Nuble, Chile, quienes mayoritariamente muestran alto nivel de satisfacción con las acciones desarrolladas. Esta intervención brinda la oportunidad de avanzar en el desarrollo de competencias técnicas, valores y actitudes del estudiantado y, junto con ello, lograr un mayor compromiso y aporte al desarrollo territorial de las regiones.

Palabras clave: Aprendizaje servicio; Competencias específicas; Competencias genéricas; Educación superior; Institucionalización; Vinculación bidireccional. 


\title{
Institutionalization of service learning in the Faculty of Business Sciences at the University of Bío-Bío: linking higher education with the local community
}

\begin{abstract}
The service-learning methodology offers countless opportunities for higher education to strengthen the teaching-learning process through the connection between students and the community. Service-learning allows extending the reference areas of the teaching work, from the classroom to the fieldwork. Thus, students can recognize the particularities of the environment and discover the needs of the local community. The aim of this study describes the actions developed to integrate and institutionalize the service-learning methodology as part of the development of its undergraduate courses in the Faculty of Business Sciences at the University of Bío-Bío of Chile. Results indicate that to date, 901 students and 19 teachers have participated, working collaboratively with community partners in training sessions and specific consultancies. Currently, 276 community partners belonging to 20 institutions in the regions of Biobío and Ñuble, Chile, have received assistance and, most of them show a high level of satisfaction with the actions developed. This methodology provides students with technical skill, value, and attitude development opportunities. Furthermore, it allows students to achieve greater commitment and contribution to the territorial development of the regions in which they study.
\end{abstract}

Keywords: Service-learning; Specific competences; Generic competences; Higher education; Institutionalization; Two-way linkage. 


\title{
Institucionalização da aprendizagem-serviço na Faculdade de Ciências Empresariais da Universidad del Bío-Bío: vinculação do ensino superior com da comunidade local
}

\begin{abstract}
Resumo
A metodologia aprendizagem-serviço oferece inúmeras oportunidades para o ensino superior a fim de reforçar o processo de ensino-aprendizagem mediante o vínculo entre os estudantes e a comunidade. Permite ampliar os âmbitos de referência do trabalho docente, passando desde a sala de aula até o trabalho de campo, o que leva a reconhecer as particularidades do contexto e descobrir as necessidades da comunidade local. O objetivo deste trabalho é descrever as ações desenvolvidas pela Faculdade de Ciências Empresariais da Universidad del Bío-Bío do Chile para incorporar e institucionalizar a metodologia aprendizagem-serviço como parte do desenvolvimento de suas disciplinas. Os resultados indicam que até o momento participaram 901 estudantes e 19 docentes, os quais trabalham de forma colaborativa com sócios comunitários em jornadas de capacitação e assessorias específicas. Já foram atendidos 276 sócios comunitários pertencentes a 20 instituições das regiões de Biobío e de Nuble, Chile, os quais em sua maioria mostram alto nível de satisfação com as ações desenvolvidas. Essa intervenção oferece a oportunidade de avançar no desenvolvimento de competências técnicas, valores e atitudes dos estudantes e, com isso, conseguir um maior comprometimento e contribuição para o desenvolvimento territorial das regiões.
\end{abstract}

Palavras-chave: aprendizagem-serviço; competências específicas; competências genéricas; ensino superior; institucionalização; vínculo bidirecional. 


\section{Introducción}

La metodología aprendizaje servicio (As) busca dar respuesta a necesidades reales de la sociedad, vinculando el aprendizaje académico con el servicio que lleva a cabo el estudiantado (Puig, 2009). La incorporación de la metodología As en la educación superior ofrece innumerables oportunidades para fortalecer la relación con la comunidad, contribuir a la formación profesional y personal del estudiantado, promover el compromiso social y potenciar el papel del docente como facilitador en el proceso enseñanza-aprendizaje, considerando que las acciones desarrolladas en terreno crean las condiciones para que el aprendizaje se haga más efectivo y más gratificante (Daura, 2011; Uruñuela, 2011).

La creciente necesidad por vincular al estudiantado universitario con la comunidad ha propiciado que, desde el año 2009, la Facultad de Ciencias Empresariales de la Universidad del Bío-Bío responda a este requerimiento a través de la implementación de una asignatura de formación integral institucional, la cual inicialmente desarrollaba temas de capacitación y asesorías en formulación proyectos, educación financiera, alfabetización digital, entre otras, a organizaciones públicas y privadas (Salazar, Vallejos, Muñoz y Lagos, 2019; Vallejos, Salazar, Muñoz, Arriagada y Roca, 2016). Lo anterior, si bien fue un aporte, requería de una sistematización y formalización de estas iniciativas, de tal manera que desde el año 2015 se puso en marcha el Plan de Mejoramiento denominado "Desarrollo de competencias del estudiantado de la Facultad de Ciencias Empresariales (FACE) a través de la metodología aprendizaje servicio (AS) y el uso de tecnologías de la información” (РМ Uвв 1402), financiado por el Banco Mundial mediante el Programa Mecesup del Ministerio de Educación de Chile (Mecesup, 2015).

Este plan de mejoramiento tiene por objetivo desarrollar las competencias profesionales en el proceso de formación de las carreras de la FACE, utilizando la metodología As, "a través de mejoras en el aprendizaje y el desarrollo de competencias y habilidades profesionales en el proceso de formación de las carreras, mediante el trabajo académico de servicio y experiencias reales en el marco del contenido de sus asignaturas" (Mecesup, 2015, p. 6). Específicamente, esta iniciativa se centra en el fortalecimiento del proceso de enseñanza-aprendizaje de los estudiantes de las carreras Contador Público y Auditor, Ingeniería Comercial, Ingeniería Civil en Informática e Ingeniería de Ejecución en Computación e Informática, potenciando sus competencias específicas y genéricas a través de la metodología As (Mecesup, 2015). 
Esto en concordancia con el propósito del modelo educativo de la Universidad, que es la formación integral del estudiantado, considerando su desarrollo como persona y como profesional en su disciplina, desde el desarrollo del conocimiento, las habilidades y las actitudes (Universidad del Bío-Bío, 2008).

El aporte de este trabajo en la implementación e institucionalización de la metodología As en la FACE radica en el desarrollo de actividades de planificación, socialización, sensibilización y visibilización de los alcances de este enfoque, así como también en la realización de un programa de capacitación para académicos y profesionales vinculados al desarrollo de las asignaturas comprometidas en el Plan de Mejoramiento чвв 1402. Esto ha dado lugar a la construcción de un primer itinerario de implementación y diseño de instrumentos de seguimiento y monitoreo de las actividades desarrolladas, especialmente para el trabajo que se realiza en terreno (Salazar et ál., 2019). Se destacan la bidireccionalidad del proceso y la posibilidad de vinculación con diversas organizaciones e instituciones locales y regionales, ya que el estudiantado realiza capacitaciones, asesorías, prácticas profesionales y actividades de titulación en una modalidad de trabajo basada en los requerimientos de socios comunitarios - tanto hombres como mujeres-, con el apoyo especializado de docentes.

Este trabajo pretende ser una inspiración para quienes deseen iniciar este proceso — no exento de dificultades—, ya sea en unidades académicas de áreas disciplinares similares o diferentes, dentro de esta u otra casa de estudios. En esta metodología, más que contar con gran cantidad de recursos económicos, se torna esencial contar con el apoyo de la unidad académica correspondiente y de los docentes a cargo de las asignaturas en que se implementa. En ese sentido, en la primera parte de este artículo se proporciona una revisión general de la literatura relativa al As, para seguidamente presentar la metodología utilizada en el levantamiento y procesamiento de la información. En la tercera parte se exhiben los principales resultados alcanzados con la implementación de la metodología As en la FACE de la Universidad del Bío-Bío y, por último, se da paso a las principales conclusiones concernientes a esta experiencia.

\section{Marco teórico}

La educación en el siglo xxi enfrenta mayores desafíos en términos de actualización de contenidos y metodologías, y en el desarrollo de competencias y habilidades. El estudiantado se encuentra con un medioambiente natural y social en constante 
transformación que lleva a enfrentar nuevos desafíos en el ámbito formativo y a demandar un nuevo tipo de educación que responda a la complejidad del mundo actual (Reimers y Chung, 2016). En este contexto, la metodología As ofrece innumerables oportunidades para actualizar enfoques y estrategias educativas en docencia universitaria, fortaleciendo la relación con la comunidad y contribuyendo a la formación profesional y personal de los estudiantes.

Para Bringle y Hatcher (1996), As es un método de aprendizaje mediante el cual el estudiantado brinda un servicio a la comunidad, lo que permite obtener una mayor comprensión de los contenidos académicos a través de la reflexión y la responsabilidad cívica. Mientras que para Eyler (2009), el As es un tipo de educación experiencial que incluye estudio académico y servicio comunitario, y que logra mejorar la calidad de ambos a través de un cambio en el paradigma educativo, con un enfoque participativo y democrático.

En general, a nivel internacional existe una definición consensuada de As a partir de tres rasgos fundamentales: se trata de un servicio solidario destinado a atender necesidades reales y sentidas de una comunidad; es protagonizado fundamentalmente por estudiantes, con la participación de docentes y representantes de entidades sociales; y es planificado de forma integrada con los contenidos curriculares y las actividades que conforman el servicio a la comunidad, a fin de mejorar la calidad de los aprendizajes escolares (Chaverri, 2015; Furco y Billig, 2002; Mayor y Rodríguez, 2015; Puig, 2009; Puig, Batlle, Bosch y Palos, 2007; Tapia, 2001; Tapia, Amar, Montes, Tapia y Yaber, 2013).

Según Rodríguez-Gallego (2014),

el modelo As constituye un ejercicio de unión de aspectos fundamentales que tradicionalmente están por separado en las instituciones educativas: la teoría con la práctica, el aula con la realidad, la formación con el compromiso y la cognición con la emoción. (p. 98)

El aprendizaje conectado con el servicio favorece la comprensión de la realidad, y se caracteriza por ser contextual, vivencial e integral (Mayor, 2019), proporcionando un cambio de paradigma educativo; desde aprendizajes teóricos y memorísticos se pasa a aprendizajes prácticos y derivados de la experiencia (aprendizaje experiencial), como lo sustentan teóricamente los escritos de Dewey y Kolb (Salazar, 2016). Es importante aclarar que Dewey (1938) y Freire (2002) ya mencionaban que la experiencia es fundamental para la educación, pero que esta no necesariamente genera los aprendizajes que se requieren, por lo mismo no debemos quedarnos únicamente 
con la experiencia vivida. Por este motivo, como lo plantea Tapia (2014), es importante definir claramente qué se entiende por As, para diferenciar esta propuesta de otro tipo de iniciativas.

De acuerdo a lo indicado por Manzano (citado en Rodríguez-Gallego, 2014), para que exista As es necesaria la estructuración simultánea de cuatro características específicas: aprendizaje académico, orientación hacia la transformación social, diálogo horizontal con la comunidad y peso presencial del estudiantado. En otras palabras, esto es la acción educativa intencionalmente planificada para dar respuesta a una necesidad real de la sociedad (Puig y Palos, 2006), gracias a la cual mejora el proceso de aprendizaje y compromiso del estudiantado a través de experiencias contextualizadas en la comunidad, mediante un proyecto estructurado y articulado con contenidos académicos, que permite aplicar lo aprendido al servicio de situaciones de la vida real (Mayor, 2018; Puig et ál., 2007; Uribe, 2018).

En síntesis, as es una actividad de utilidad social y significativa para el conjunto de protagonistas, integrada al contenido curricular, que toma distancia de debates teóricos sin aplicación práctica (Mayor, 2019). La intensidad y duración del servicio se planifica en función de alcanzar los objetivos educativos y comunitarios propuestos, procurando impactar positivamente en la sociedad civil (Rodríguez-Gallego, 2014; Tapia, 2010).

En el estudiantado, los proyectos de as estimulan el aprendizaje basado en el protagonismo activo, combinando los contenidos curriculares con el compromiso cívico y una conciencia ética-solidaria, lo que permite la implicación en las necesidades del entorno con la finalidad de mejorarlo, a la vez que se desarrollan valores de solidaridad, responsabilidad y participación ciudadana (Jacoby, 1996; Mayor, 2013; Ochoa y Pérez, 2019; Páez y Puig, 2013; Pérez y Ochoa, 2017; Puig y Palos, 2006; Sandrea y Reyes, 2010; Tapia, 2010). Se trata, entonces, de fomentar una metodología motivadora para la aplicación de conocimientos adquiridos en el aula, con miras a generar nuevas competencias relevantes para el desarrollo profesional y personal (Ferrán-Zubillaga y Guinot-Viciano, 2012; Tapia, Bridi, Maidana y Rial, 2015).

En este sentido, los actores de estos proyectos poseen un papel fundamental: el docente cumple el cargo de facilitador, convirtiéndose así en un agente motivador más que en un mero trasmisor de información (Godoy-Pozo, Illesca-Pretty, SeguelPalma y Salas-Quijada, 2019). Debe incentivar en el estudiantado el pensamiento crítico y la responsabilidad cívica, priorizando el aprendizaje construido en necesidades reales por sobre las clases teórico-expositivas desconectadas del entorno (Francisco y Moliner, 2010; Martínez, 2010). Los participantes de la comunidad se 
involucran también activamente en la formulación de la propuesta de As, identificando necesidades reales y no hipotéticas (Furco, 2002; Martínez-Odria, 2007). En el estudiantado, las investigaciones indican que el As favorece la adquisición de conocimientos disciplinares, habilidades de liderazgo, valores como la justicia social; impacta en el desarrollo de actitudes altruistas, compromiso cívico y responsabilidad social (Cooper, Cripps y Reisman, 2013; Groh, Stallwood y Daniels, 2011; Jenkins y Sheehey, 2011); promueve la participación colaborativa de estudiantes y docentes (Mayor y Rodríguez, 2017); permite que el estudiante reconozca las necesidades de los destinatarios del servicio e impulsa el aprendizaje con sentido cívico, social, ético y moral (Puig, Gijón, Martín y Rubio, 2011); incrementa las habilidades de trabajo en equipo, comunicación, responsabilidad social, solidaridad y creatividad (Rodríguez, 2014). Los ámbitos beneficiados con los proyectos de AS son mayormente tres: 1) currículum académico, al reelaborar contenidos teóricos, haciéndolos pertinentes para un contexto social; 2) formación en valores, al educar para la ciudadanía; y 3) vinculación con la comunidad, al promover la intervención social (Rodríguez, 2014).

En los proyectos de As, la universidad se convierte en un espacio de participación que entra en diálogo con la comunidad, en una relación recíproca que permite fortalecer el tejido social para responder a los desafíos educativos que plantea la sociedad actual (Escofet, Folgueiras, Luna y Palou, 2016). En tal sentido, el modelo de formación de la universidad y su misión educativa deben redefinirse para incorporar en su quehacer espacios de convivencia y prácticas educativas con implicaciones en la comunidad, pues el propósito ahora debe estar enfocado en aplicar el conocimiento a las necesidades de la sociedad (Mayor y Rodríguez, 2015; Rodríguez, 2014; Salazar, 2016).

Existen dos formas de implementar la metodología As en la universidad: 1) la forma curricular, mediante la inserción de la metodología en el currículum profesional; y 2) la forma cocurricular, en la que la metodología se inserta como una actividad de extensión o curso optativo (Jenkins y Sheehey, 2011; Jouannet, Salas y Contreras, 2013). Las instituciones pueden comenzar a desarrollar proyectos con rasgos de As, que con el tiempo pueden tener continuidad, vinculándose con una cátedra y generando actividades más complejas que culminen en procesos de institucionalización de programas de As (Tapia, 2010).

Bringle y Hatcher (1996) proponen un modelo de desarrollo de As a nivel institucional en el que se identifican cuatro componentes principales del proceso de implementación: la institución, la facultad, el estudiantado y la comunidad, para cada uno de los cuales se incluye una secuencia de pasos o acciones. En este sentido, el 
libro Aprendizaje Servicio en la Educación Superior Chilena (Pizarro y Hasbún, 2019) muestra una radiografía de las experiencias a nivel nacional, dando cuenta de cómo fueron sus inicios, detallando cuáles universidades se encuentran en etapa de institucionalización, de construcción de calidad o de creación de masa crítica. Por su parte, esta experiencia pretende avanzar hacia la institucionalización de la metodología, a partir de una implementación replicable y factible de poner en funcionamiento toda la institución desde la experiencia de una facultad (Salazar et ál., 2019).

Respecto a experiencias en Latinoamérica, la Universidad Católica de Chile ha diseñado un modelo de institucionalización que pretende incorporar "la metodología A+S en toda la universidad a nivel curricular de pregrado de manera sostenible. El modelo diseñado busca lograr las tres condiciones de integración institucional —estructural, cultural y procedimental - de la metodología A+S a nivel de sus carreras" (Jouannet et ál., 2015, p. 1).

\section{Metodología}

Este trabajo corresponde a un estudio de caso (Stake, 2007) con enfoque cuantitativo y alcance descriptivo de corte longitudinal (Hernández, Fernández y Baptista, 2010), que busca describir las acciones desarrolladas como parte de la implementación de la metodología As en la FACE de la Universidad del Bío-Bío, durante el periodo 2016-2018.

Dicha propuesta metodológica de incorporación de As en una asignatura de la FACE contempla un itinerario que abarca las etapas de planificación, implementación y evaluación. Estas etapas están acompañadas de un conjunto de herramientas e instrumentos para registrar y recopilar información, que se transforman en una fuente de información continua que apoya el desarrollo de actividades y facilita la toma de decisiones a través del registro de hechos y datos.

La etapa de planificación parte al término de cada año y se extiende hasta el inicio del primer semestre académico. Consiste en el levantamiento de información sobre las necesidades y problemáticas de la comunidad, el análisis de las asignaturas para identificar la que puede incorporar el trabajo colaborativo con la comunidad y la organización, junto con los docentes de la actividad o actividades por desarrollar dentro de la asignatura. Esta etapa considera además la socialización de la metodología, por lo tanto, implica realizar un taller de inducción dirigido a docentes para explicarla y motivar su utilización, y otro dirigido al estudiantado. En este se aborda la metodología y sus implicancias, se forman los equipos de trabajo, distribuyendo 
los cargos que cumplirá cada estudiante; se aplica la estrategia de reflexión inicial, y se exponen las principales habilidades sociales por desarrollar para el trabajo con otros, aspecto que, por cierto, se ha evidenciado como una de las principales debilidades del estudiantado en la puesta en marcha de las actividades de capacitación y asesorías planificadas para responder a los requerimientos. Este taller se realiza en la primera sesión de clases (con una duración de dos horas pedagógicas) del periodo que comprende la incorporación de la metodología As en una asignatura, marcando así el inicio de esta experiencia.

La etapa de implementación corresponde al trabajo desarrollado por el estudiantado para responder al servicio o producto comprometido, según lo solicitado por socios comunitarios, bajo la guía y supervisión del docente a cargo de la asignatura. Antes de iniciar el servicio se desarrolla una actividad de acercamiento que consiste en hacer el primer contacto del docente o de los estudiantes con los socios comunitarios. La duración de esta etapa varía de acuerdo a la naturaleza del servicio: en general, cuando el estudiantado brinda capacitaciones, este se prepara durante dos semanas para, posteriormente, realizar tres o cuatro sesiones (de aproximadamente dos horas y media) de trabajo con los socios comunitarios; cuando efectúa asesorías directas, el servicio puede durar todo el semestre.

La tercera y última etapa, la evaluación, considera la reflexión con el estudiantado sobre los aprendizajes logrados, mediante un cierre de la actividad con la participación de todos los actores involucrados, en la que se aplican los cuestionarios de apreciación que permiten recoger información para evaluar el proceso en general y determinar el nivel de satisfacción de los participantes. Este taller de reflexión final se desarrolla tras finalizar el servicio, en una sesión de clases (de dos horas pedagógicas). La información se puede recoger de diferentes maneras, dependiendo de la estrategia de reflexión utilizada. No obstante, en cualquier caso, corresponde a información directamente escrita o verbalizada por los mismos estudiantes respecto a los principales aprendizajes y vivencias experimentadas en esta actividad, que posteriormente se tabula y sintetiza.

Para recabar la información relativa a la percepción que tienen las personas involucradas respecto a la implementación de la metodología, el equipo del Proyecto Aprendizaje Servicio emplea un cuestionario de apreciación que ha diseñado y validado, con el objetivo de que cada parte (estudiantes, docentes y socios comunitarios) evalúe el trabajo. Los estudiantes evalúan el suyo propio en la asignatura; y los docentes, el cumplimento de los resultados de aprendizaje y la implementación de la metodología en su asignatura. El cuestionario se aplica a los socios comunitarios 
para evaluar su nivel de satisfacción con el trabajo colaborativo desarrollado, así como la calidad y pertinencia del servicio recibido.

Como vemos, el instrumento tiene una versión distinta para cada actor, aunque todas se miden con una escala tipo Likert de siete puntos, donde 1 se asigna al valor más bajo y 7 al más alto, para evaluar qué tan de acuerdo o satisfecho — según corresponda - se encuentra con cada aspecto consultado. En el caso de la versión destinada a estudiantes, el cuestionario consta de 17 ítems, un apartado para opiniones finales y sugerencias, y una tercera sección de datos básicos de identificación de la asignatura y del estudiante; se consulta: carrera, sede, curso o nivel, docente de la asignatura, fecha, sexo y edad. Por su parte, la versión para el docente está dividida en cuatro secciones: la primera consta de 12 ítems con los aspectos a evaluar; la segunda es para opiniones finales y sugerencias; la tercera para proporcionar las fortalezas y debilidades del trabajo con la metodología As y, por último, una sección en la que se solicita la identificación de la asignatura: carrera, nombre de la asignatura, curso o nivel, sede y fecha. En cuanto a la versión destinada a los socios comunitarios beneficiarios de las asignaturas implementadas con metodología As, la constituyen tres secciones: la primera con 13 ítems a evaluar; la segunda para opiniones finales y sugerencias, y la tercera parte identificar carrera, asignatura, curso o nivel, fecha y actividad de cada socio comunitario. Cabe señalar que este instrumento ha tenido constantes mejoras y actualizaciones a partir del propio aprendizaje alcanzado entre los años 2016 y 2018.

Los instrumentos de recolección de información se aplican semestralmente al estudiantado, los docentes y socios comunitarios al finalizar la implementación de la asignatura con As, y puede ser de manera presencial o a través de correo electrónico. El estudiantado diligencia este cuestionario en la actividad de reflexión final (descrita anteriormente); los socios comunitarios, en la actividad de cierre de la asignatura; y los docentes a cargo de la asignatura, al cierre de esta.

El cuestionario lo contestaron 750 personas, que corresponden al 62,1 \% de los participantes históricos en el Proyecto Aprendizaje Servicio de la FACE (tabla 1). La muestra fue no probabilística por conveniencia, siendo el criterio de selección la participación en una asignatura con metodología As. No obstante, la respuesta a este cuestionario es voluntaria para todos los actores involucrados. Cabe aclarar que si el docente de la asignatura es un miembro del equipo coordinador, no necesita responder el cuestionario, dado que se busca identificar fortalezas y debilidades de las actividades realizadas con metodología As para considerar estos insumos en el proceso de mejora continua de esta iniciativa. 
Tabla 1. Población y muestra del cuestionario de percepción de las asignaturas con As durante el periodo 2016-2018.

\begin{tabular}{|l|c|c|c|}
\cline { 2 - 4 } \multicolumn{1}{c|}{} & Población & Muestra & Proporción \\
\hline Estudiantes & 901 & 608 & $67,5 \%$ \\
\hline $\begin{array}{l}\text { Docentes (considera número de participaciones, } \\
\text { no personas únicas) }\end{array}$ & 31 & 12 & $38,7 \%$ \\
\hline Socios comunitarios & 276 & 130 & $47,1 \%$ \\
\hline Total & $\mathbf{1 2 0 8}$ & $\mathbf{7 5 0}$ & $\mathbf{6 2 , 1} \%$ \\
\hline
\end{tabular}

Fuente: Elaboración propia a partir de los datos del Proyecto Aprendizaje Servicio de la FACE.

El análisis de los datos y sistematización de la información recopilada durante el periodo 2016-2018 se efectuó a través de herramientas propias de la estadística descriptiva, en este caso el software Microsoft Excel.

\section{Resultados}

\section{Implementación de la metodología As}

La propuesta metodológica de trabajo para la implementación de la metodología As en las carreras de Ingeniería Comercial, Contador Público y Auditor, Ingeniería Civil en Informática e Ingeniería de Ejecución en Computación e Informática de la FACE contempla el desarrollo de un modelo de implementación construido, utilizando como referencia otros modelos instaurados en universidades chilenas (Jouannet et ál., 2015, 2013). Este modelo se conforma de un itinerario con tres etapas claves: planificación, implementación y evaluación de la metodología, las cuales van acompañadas de una serie de instrumentos relevantes para recopilar información de utilidad, tanto para los docentes como para el estudiantado, con el fin de organizar, registrar, sistematizar y monitorear el trabajo a desarrollar en terreno.

Esta experiencia se ha centrado en los siguientes ejes de desarrollo: socialización de la metodología al interior de la Facultad y de la Institución; capacitación docente en metodología As; visibilización en medios de comunicación internos y externos; y la generación de redes con diferentes instituciones.

La implementación de esta metodología comenzó formalmente el primer semestre del año 2016, y al año 2018 ha sido incorporada en 29 cursos de las carreras 
de esta facultad (tabla 2), contando con la participación de 19 docentes diferentes, además de su integración en prácticas profesionales y actividades de titulación de estas carreras.

En este sentido, es pertinente indicar que uno de los aspectos fundamentales, si no el más importante, es la participación y compromiso de los docentes que trabajan directamente en las asignaturas, lo cual resulta primordial para la ejecución de la metodología As con rigurosidad y eficacia. Para ello, los docentes deben estar involucrados desde la génesis de la iniciativa y el convencimiento debe ser natural, ya que esta requiere de más tiempo y dedicación de lo habitual para su ejecución, tanto para docentes como para estudiantes. De no ser así, pueden surgir problemas en la implementación de esta metodología, dado que el tiempo de dedicación adicional de los docentes no siempre se refleja de manera explícita en su carga académica, ni cuentan necesariamente con el apoyo de procedimientos administrativos internos, como por ejemplo: ayudantes adicionales, más tiempo para el desarrollo de las asignaturas o liberación de tiempo de otras actividades, entre otros aspectos, lo cual intensifica la necesidad de institucionalización y definición de políticas universitarias que hagan sustentable esta iniciativa en el tiempo. Esta experiencia en particular (focalizada en una facultad) puede dar cuenta de ello, dado que hay iniciativas y apoyos que escapan a sus atribuciones y que requieren necesariamente de la intervención desde la institucionalidad de la universidad.

Tabla 2. Número de asignaturas implementadas con AS en la FACE durante el periodo 2016-2018.

\begin{tabular}{|l|c|c|c|c|}
\hline \multicolumn{1}{|c|}{ Carrera } & $\mathbf{2 0 1 6}$ & $\mathbf{2 0 1 7}$ & $\mathbf{2 0 1 8}$ & Total \\
\hline Ingeniería Comercial & 7 & 2 & 4 & 13 \\
\hline Contador Público y Auditor & 2 & 6 & 5 & 13 \\
\hline Ingeniería Civil en Informática & 0 & 2 & 1 & 3 \\
\hline Total & 9 & 10 & 10 & 29 \\
\hline
\end{tabular}

Fuente: elaboración propia a partir de los datos del Proyecto Aprendizaje Servicio de la FACE.

En estas asignaturas, se han atendido a 276 personas beneficiarias durante el periodo 2016-2018, a partir del trabajo del estudiantado de las carreras de Ingeniería Comercial, Contador Público y Auditor e Ingeniería Civil en Informática de la FACE (tabla 3). Cabe señalar que la cantidad potencial de socios comunitarios por atender se define de acuerdo a la naturaleza del servicio brindado. 
Tabla 3. Número de socios comunitarios atendidos en las asignaturas implementadas con As de la FACE durante el periodo 2016-2018.

\begin{tabular}{|l|c|c|c|c|}
\hline \multicolumn{1}{|c|}{ Carrera } & $\mathbf{2 0 1 6}$ & $\mathbf{2 0 1 7}$ & $\mathbf{2 0 1 8}$ & Total \\
\hline Ingeniería Comercial & 43 & 11 & 36 & 90 \\
\hline Contador Público y Auditor & 17 & 57 & 72 & 146 \\
\hline Ingeniería Civil en Informática & 0 & 39 & 1 & 40 \\
\hline Total & $\mathbf{6 0}$ & $\mathbf{1 0 7}$ & $\mathbf{1 0 9}$ & $\mathbf{2 7 6}$ \\
\hline
\end{tabular}

Fuente: elaboración propia a partir de los datos del Proyecto Aprendizaje Servicio de la FACE.

Estos socios comunitarios pertenecen a veinte organizaciones públicas y privadas de las regiones de Nuble y del Biobío, con las cuales se han establecido acuerdos de trabajo colaborativo (tabla 4). En algunas ocasiones, se trabaja directamente con la institución en la resolución de algún proyecto en particular; en otras, con los usuarios de estas organizaciones.

Asimismo, existen instituciones con las que se trabaja anualmente, propiciando acuerdos de trabajo colaborativo, que se reflejan en un número mucho mayor de usuarios atendidos, mientras que en otras ocasiones el trabajo se ha desarrollado de manera puntual. Todo esto se explica, principalmente, por la demanda que es posible atender semestralmente, de acuerdo con la capacidad instalada en la facultad.

Tabla 4. Número de socios comunitarios institucionales y de socios comunitarios atendidos durante el periodo 2016-2018.

\begin{tabular}{|c|l|c|}
\hline N. ${ }^{\circ}$ & \multicolumn{1}{|c|}{ Socio comunitario institucional } & $\begin{array}{c}\text { Cantidad de } \\
\text { beneficiarios }\end{array}$ \\
\hline 1 & Consultora Sur Social - Concepción & 1 \\
\hline 2 & $\begin{array}{l}\text { Fundación Trabajo para un Hermano Concepción (TPH) } \\
\text { - Concepción }\end{array}$ & 55 \\
\hline 3 & $\begin{array}{l}\text { Instituto de Formación y Capacitación Popular (Infocap) } \\
\text { - Concepción }\end{array}$ & 3 \\
\hline 4 & Ilustre Municipalidad de Coihueco & 1 \\
\hline 5 & Ilustre Municipalidad de Pemuco & 1 \\
\hline 6 & Ilustre Municipalidad de Pinto & 1 \\
\hline 7 & Ilustre Municipalidad de Quillón & 1 \\
\hline
\end{tabular}


Institucionalización del aprendizaje servicio en la Facultad de Ciencias Empresariales...

\begin{tabular}{|c|c|c|}
\hline N.o & Socio comunitario institucional & $\begin{array}{l}\text { Cantidad de } \\
\text { beneficiarios }\end{array}$ \\
\hline 8 & Ilustre Municipalidad de Ránquil & 1 \\
\hline 9 & Ilustre Municipalidad de San Ignacio & 1 \\
\hline 10 & Ilustre Municipalidad de Trehuaco & 1 \\
\hline 11 & $\begin{array}{l}\text { Oficina de Fomento Productivo Dirección de Desarrollo Comunitario } \\
\text { - Ilustre Municipalidad de Chillán }\end{array}$ & 12 \\
\hline 12 & Programa de Desarrollo Local (Prodesal) - San Ignacio & 25 \\
\hline 13 & $\begin{array}{l}\text { Programa Mujer, Asociatividad y Emprendimiento Sernameg } \\
\text { - Chillán }\end{array}$ & 10 \\
\hline 14 & $\begin{array}{l}\text { Programa Mujer, Asociatividad y Emprendimiento Sernameg } \\
\text { - Concepción }\end{array}$ & 8 \\
\hline 15 & $\begin{array}{l}\text { Programa Mujeres Jefas de Hogar Sernameg - Ilustre Municipalidad } \\
\text { de Chillán }\end{array}$ & 11 \\
\hline 16 & $\begin{array}{l}\text { Programa Mujeres Jefas de Hogar Sernameg - Ilustre Municipalidad } \\
\text { de Cabrero }\end{array}$ & 3 \\
\hline 17 & $\begin{array}{l}\text { Programa Mujeres Jefas de Hogar Sernameg - Ilustre Municipalidad } \\
\text { de Chillán Viejo }\end{array}$ & 109 \\
\hline 18 & $\begin{array}{l}\text { Programa Mujeres Jefas de Hogar Sernameg - Ilustre Municipalidad } \\
\text { de Quillón }\end{array}$ & 24 \\
\hline 19 & Servicio de Salud Biobío - Los Ángeles & 1 \\
\hline \multirow[t]{2}{*}{20} & $\begin{array}{l}\text { Servicio Nacional de la Mujer y Equidad de Género (Sernameg) } \\
\text { - Concepción }\end{array}$ & 7 \\
\hline & Total & 276 \\
\hline
\end{tabular}

Fuente: elaboración propia a partir de los datos del Proyecto Aprendizaje Servicio de la FACE.

A su vez, en estas asignaturas han participado 901 estudiantes de las sedes de Chillán y Concepción de la FACE (tabla 5), que son los responsables de desarrollar los diferentes servicios comprometidos en estas intervenciones. El trabajo se ha realizado con estudiantes de todos los niveles, adecuándolo según el año de la carrera que están cursando. Además, se promueve el trabajo en equipo, con funciones y responsabilidades claramente establecidas para cada integrante desde el inicio de la actividad. 
Tabla 5. Número de estudiantes que participaron en las asignaturas implementadas con As de la FACE durante el periodo 2016-2018.

\begin{tabular}{|l|c|c|c|c|}
\hline \multicolumn{1}{|c|}{ Carrera } & $\mathbf{2 0 1 6}$ & $\mathbf{2 0 1 7}$ & $\mathbf{2 0 1 8}$ & Total \\
\hline Ingeniería Comercial & 187 & 46 & 149 & 382 \\
\hline Contador Público y Auditor & 77 & 193 & 162 & 432 \\
\hline Ingeniería Civil en Informática & 0 & 66 & 21 & 87 \\
\hline Total & $\mathbf{2 6 4}$ & $\mathbf{3 0 5}$ & $\mathbf{3 3 2}$ & $\mathbf{9 0 1}$ \\
\hline
\end{tabular}

Fuente: elaboración propia a partir de los datos del Proyecto Aprendizaje Servicio de la FACE.

En estas asignaturas, el estudiantado ha elaborado diversas capacitaciones y asesorías, según se detalla en la tabla 6. Este trabajo, entre otras cosas, ha permitido desarrollar una aplicación móvil, lograr la adjudicación de proyectos del fondo de capital semilla de algunos municipios para fortalecer e impulsar iniciativas de negocio lideradas por mujeres, y en general, entregar herramientas específicas que permitan a las personas microemprendedoras fortalecer sus iniciativas de negocios.

Tabla 6. Resumen de los servicios proporcionados durante el periodo 2016-2018.

\begin{tabular}{|c|c|}
\hline Carrera & Servicio realizado \\
\hline \multirow{7}{*}{ Ingeniería Comercial } & - Asesoría en plan de marketing y estudio de mercado. \\
\hline & - Asesoría en análisis de costos y diseño de sistema de costeo. \\
\hline & $\begin{array}{l}\text { - Elaboración de proyectos sociales de acuerdo a la propuesta por el } \\
\text { Ministerio de Desarrollo Social y Subdere (marco lógico). }\end{array}$ \\
\hline & - Desarrollo o mejora del modelo de negocio (método Canvas). \\
\hline & $\begin{array}{l}\text { - Apoyo en la formulación y postulación de proyectos a fondos } \\
\text { concursables. }\end{array}$ \\
\hline & - Capacitación en alfabetización digital. \\
\hline & - Control de ingresos y egresos con apoyo de planillas Excel. \\
\hline \multirow{4}{*}{$\begin{array}{l}\text { Contador Público y } \\
\text { Auditor }\end{array}$} & - Elaboración de presupuestos. \\
\hline & - Capacitación en educación financiera. \\
\hline & $\begin{array}{l}\text { - Capacitación en uso de herramientas digitales y redes sociales para } \\
\text { comercialización de productos. }\end{array}$ \\
\hline & - Asesoría en sistema contable y régimen tributario. \\
\hline
\end{tabular}


Institucionalización del aprendizaje servicio en la Facultad de Ciencias Empresariales...

\begin{tabular}{|c|c|}
\hline Carrera & Servicio realizado \\
\hline \multirow{6}{*}{$\begin{array}{l}\text { Contador Público y } \\
\text { Auditor }\end{array}$} & $\begin{array}{l}\text { - Asesoría en inicio de actividades y timbraje de formularios, } \\
\text { elaboración de contratos, declaraciones previsionales, formulario IVA y } \\
\text { liquidaciones de sueldo. }\end{array}$ \\
\hline & $\begin{array}{l}\text { - Asesoría en elaboración de estados finales (balance y estado de } \\
\text { resultado). }\end{array}$ \\
\hline & $\begin{array}{l}\text { - Capacitación en elaboración de costos y uso de planilla Excel para el } \\
\text { registro de gastos e ingresos. }\end{array}$ \\
\hline & - Capacitación en regímenes tributarios y documentación electrónica. \\
\hline & - Capacitación en contabilidad básica y formalización tributaria. \\
\hline & - Capacitación en costos y uso de planillas Excel. \\
\hline \multirow{2}{*}{$\begin{array}{l}\text { Ingeniería Civil en } \\
\text { Informática }\end{array}$} & - Capacitación uso de redes sociales y planillas de cálculo Excel. \\
\hline & - Desarrollo de aplicación para dispositivos móviles. \\
\hline
\end{tabular}

Fuente: elaboración propia a partir de los datos del Proyecto Aprendizaje Servicio de la FACE.

Las experiencias descritas permiten constatar que el As ofrece una oportunidad para innovar en educación, en la medida en que constituye una metodología vivencial, que contribuye a la búsqueda de soluciones a diversos problemas o necesidades de la comunidad con excelencia, solidaridad, equidad y respeto, fortaleciendo el aprendizaje del estudiantado, el compromiso de los docentes y la participación de la sociedad civil en la promoción del desarrollo sustentable.

Es tentador observar los resultados en términos macro; las cantidades de estudiantes, profesores y socios comunitarios pueden considerarse dato suficiente en comparación con las dificultades encontradas en el camino. Pero la experiencia de este proyecto ha puesto el énfasis en el proceso, tomando especial relevancia la consecución efectiva de los resultados, más allá de las cifras por sí mismas. Para ello ha sido significativo contar con reuniones permanentes, espacios de consultas, más el trabajo de reflexión con el estudiantado, la asistencia a presentaciones y entrevistas entre estudiantes y socios comunitarios, y la retroalimentación proporcionada; todos temas demandantes que significan mucho tiempo y dedicación, pero que en definitiva corresponden a los resultados más valiosos de cada experiencia desarrollada.

La implementación de esta metodología ha permitido la articulación con otras facultades de la Universidad del Bío-Bío, para dar respuesta a requerimientos de 
diversos tipos que exceden el campo del conocimiento de las Ciencias Empresariales. Tal es el caso del trabajo desarrollado con la carrera de Ingeniería en Alimentos de la Facultad de Ciencias de la Salud y de los Alimentos; este esfuerzo colaborativo permitió establecer el Programa de Capacitación en Manipulación de Alimentos para mujeres de los municipios de Chillán y Chillán Viejo, adscritas al programa Mujeres Jefas de Hogar del Servicio Nacional de la Mujer y la Equidad de Género. Todas estas actividades han incluido un importante y significativo trabajo en terreno, que ha posibilitado que el estudiantado conozca comunas con un alto porcentaje de población rural como San Ignacio, El Carmen y Quillón, y que se relacionen con instituciones públicas y privadas vinculadas al fomento productivo y el desarrollo local.

Uno de los resultados obtenidos está relacionado con el trabajo en terreno y con la propuesta de intervención de la metodología As en territorios en transformación, bajo la perspectiva urbano y rural, con población en condiciones de vulnerabilidad, lo cual reafirma la relevancia de la labor de las instituciones de educación para aportar, en conjunto con actores económicos, políticos y sociales, en la generación de iniciativas de desarrollo local con un enfoque integrador que responda a las particularidades y potencialidades del territorio.

Además, fruto de estas experiencias se ha constituido un equipo multidisciplinario de trabajo, que ha sido fundamental para la implementación de las mismas. Este se concentra en las tareas de gestión, implementación, vinculación con el medio y acompañamiento permanente a los docentes en temas pedagógicos, operacionales e informáticos asociados al desarrollo de la metodología en una asignatura, así como todo el seguimiento administrativo necesario para su funcionamiento.

\section{Percepción de estudiantes, docentes y socios comunitarios}

La tabla 7 exhibe los principales resultados obtenidos durante el periodo 2016-2018 en la escala de percepción aplicada al estudiantado; en esta se destaca la satisfacción general de los estudiantes con respecto a la participación en las asignaturas implementadas con metodología As, que mayoritariamente fueron calificadas con nota de 5 a 7. Asimismo, evaluaron positivamente la relevancia de esta experiencia para su formación profesional y personal, el ambiente de trabajo junto al socio comunitario y la metodología utilizada para aplicar los contenidos en el aula. En general, el estudiantado mostró interés en este trabajo una vez que se sintió involucrado y observó el impacto que este tiene en las demás personas y por supuesto en ellos mismos, a pesar de que en algunas ocasiones se presentaron dificultades en la comunicación con sus socios comunitarios, o con la información que estos les proporcionaron. 
Producto de la naturaleza de este tipo de experiencias y de las implicaciones del trabajo con las personas y con sus contextos específicos, el resultado del trabajo no ha sido equivalente para todos los equipos en algunas asignaturas; existen grupos que tiene dificultades con la comunicación y con el logro de los objetivos propuestos en la intervención. Esto ha afectado el nivel de satisfacción general sobre la incorporación de la metodología As, respecto de la percepción de sus compañeros. Tras la identificación de algunas dificultades en la comunicación entre el estudiantado y los socioscomunitarios, surgió la necesidad de realizar un taller introductorio en habilidades sociales. Aunque las dificultades en la interacción con los interlocutores son el resultado de los problemas propios del contexto en el que se realiza el servicio, esto no puede ser un impedimento para que el estudiantado sea incapaz de flexibilizar su conducta frente a estas situaciones y personas.

Tabla 7. Percepción del estudiantado respecto la implementación de la metodología As durante el periodo 2016-2018.

\begin{tabular}{|l|c|c|c|c|c|c|c|c|}
\cline { 2 - 7 } \multicolumn{1}{l|}{} & $\mathbf{7}$ & $\mathbf{6}$ & $\mathbf{5}$ & $\mathbf{4}$ & $\mathbf{3}$ & $\mathbf{2}$ & $\mathbf{1}$ & Total \\
\hline $\begin{array}{l}\text { Las actividades de la } \\
\text { metodología As desarrolladas } \\
\text { con la persona socia } \\
\text { comunitaria fueron relevantes } \\
\text { para su formación profesional } \\
\text { y personal. }\end{array}$ & $39,9 \%$ & $31,6 \%$ & $15,8 \%$ & $6,1 \%$ & $2,5 \%$ & $2,0 \%$ & $2,1 \%$ & $100 \%$ \\
\hline $\begin{array}{l}\text { La relación con la persona } \\
\text { socia comunitaria el/la socio/a } \\
\text { comunitario/a se desarrolló en } \\
\text { un ambiente de respeto mutuo. }\end{array}$ & $75,6 \%$ & $13,8 \%$ & $4,9 \%$ & $2,5 \%$ & $1,3 \%$ & $0,7 \%$ & $1,2 \%$ & $100 \%$ \\
\hline $\begin{array}{l}\text { La metodología As fue útil } \\
\text { para aplicar los aprendizajes } \\
\text { obtenidos en el aula. }\end{array}$ & $32,0 \%$ & $34,8 \%$ & $17,3 \%$ & $9,9 \%$ & $2,3 \%$ & $0,5 \%$ & $3,1 \%$ & $100 \%$ \\
\hline $\begin{array}{l}\text { El trabajo realizado mediante } \\
\text { metodología As es una } \\
\text { experiencia recomendable para } \\
\text { otros estudiantes. }\end{array}$ & $46,0 \%$ & $24,1 \%$ & $12,5 \%$ & $7,6 \%$ & $3,8 \%$ & $1,6 \%$ & $4,4 \%$ & $100 \%$ \\
\hline $\begin{array}{l}\text { Su grado de satisfacción } \\
\text { general con la asignatura } \\
\text { implementada con } \\
\text { metodología As.* }\end{array}$ & $29,5 \%$ & $32,5 \%$ & $19,3 \%$ & $8,9 \%$ & $3,8 \%$ & $2,0 \%$ & $4,0 \%$ & $100 \%$ \\
\hline
\end{tabular}

Nota: 7 = Totalmente de acuerdo; 6 = De acuerdo; 5 = Moderadamente de acuerdo; 4 = Ni de acuerdo ni en desacuerdo; $3=$ Moderadamente en desacuerdo; 2 = En desacuerdo; 1 = Totalmente en desacuerdo. En el caso del ítem señalado con $\left(^{*}\right): 7$ = Muy satisfecho; 6 = Satisfecho; 5 = Moderadamente satisfecho; 4 = Ni satisfecho ni insatisfecho; 3 = Moderadamente insatisfecho; 2 = Insatisfecho; $1=$ Muy insatisfecho.

Fuente: elaboración propia a partir de los datos del Proyecto Aprendizaje Servicio de la FACE. 
El 82,3\% de los socios comunitarios ha evaluado con la máxima calificación su nivel de satisfacción frente a la experiencia de participar en una asignatura implementada con la metodología As en la FACE. Al mismo tiempo, indicaron que volverían a participar en este tipo de iniciativas y manifestaron que el producto entregado por el estudiantado estuvo de acuerdo con sus necesidades (tabla 8). En general, los socios comunitarios dieron cuenta de la importancia que tienen las herramientas proporcionadas en el proyecto, además de las expectativas sobre el trabajo de la Universidad con la comunidad y su desarrollo de manera permanente.

Tabla 8. Percepción de socios comunitarios respecto la implementación de la metodología As durante el periodo 2016-2018.

\begin{tabular}{|l|c|c|c|c|c|c|c|c|}
\cline { 2 - 8 } \multicolumn{1}{l|}{} & $\mathbf{7}$ & $\mathbf{6}$ & $\mathbf{5}$ & $\mathbf{4}$ & $\mathbf{3}$ & $\mathbf{2}$ & $\mathbf{1}$ & Total \\
\hline $\begin{array}{l}\text { El producto final entregado por } \\
\text { el estudiantado está de acuerdo } \\
\text { con sus necesidades. }\end{array}$ & $77,7 \%$ & $20,0 \%$ & $1,5 \%$ & $0 \%$ & $0 \%$ & $0,8 \%$ & $0 \%$ & $100 \%$ \\
\hline $\begin{array}{l}\text { Participaría nuevamente } \\
\text { en actividades del Proyecto } \\
\text { Aprendizaje Servicio con } \\
\text { estudiantes de la ubB. }\end{array}$ & $87,7 \%$ & $10,0 \%$ & $1,5 \%$ & $0 \%$ & $0 \%$ & $0 \%$ & $0,8 \%$ & $100 \%$ \\
\hline $\begin{array}{l}\text { El equipo de estudiantes } \\
\text { mostró compromiso con el } \\
\text { trabajo desarrollado. }\end{array}$ & $83,1 \%$ & $14,6 \%$ & $1,5 \%$ & $0 \%$ & $0 \%$ & $0,8 \%$ & $0 \%$ & $100 \%$ \\
\hline $\begin{array}{l}\text { Su grado de satisfacción } \\
\text { general con esta experiencia*. }\end{array}$ & $82,3 \%$ & $16,2 \%$ & $0,8 \%$ & $0 \%$ & $0 \%$ & $0,8 \%$ & $0 \%$ & $100 \%$ \\
\hline
\end{tabular}

Nota: 7 = Totalmente de acuerdo; 6 = De acuerdo; 5 = Moderadamente de acuerdo; 4 = Ni de acuerdo ni en desacuerdo; 3 = Moderadamente en desacuerdo; 2 = En desacuerdo; 1 = Totalmente en desacuerdo. En el caso del ítem señalados con $(*): 7$ = Muy satisfecho; 6 = Satisfecho; 5 = Moderadamente satisfecho; 4 = Ni satisfecho ni insatisfecho;

3 = Moderadamente insatisfecho; 2 = Insatisfecho; $1=$ Muy insatisfecho.

Fuente: elaboración propia a partir de los datos del Proyecto Aprendizaje Servicio de la FACE.

Los docentes que han incorporado la metodología As señalan que esta experiencia es positiva para el desarrollo de su actividad y la de sus estudiantes; así mismo, destacan el hecho de que gracias a esta es posible poner en práctica los aprendizajes de la asignatura a la hora de desarrollar el producto o servicio comprometido (tabla 9). 
Tabla 9. Percepción de los docentes respecto la implementación de la metodología As durante el periodo 2016-2018.

\begin{tabular}{|l|c|c|c|c|c|c|c|c|}
\cline { 2 - 8 } \multicolumn{1}{l|}{} & $\mathbf{7}$ & $\mathbf{6}$ & $\mathbf{5}$ & $\mathbf{4}$ & $\mathbf{3}$ & $\mathbf{2}$ & $\mathbf{1}$ & Total \\
\hline $\begin{array}{l}\text { Los aprendizajes desarrollados } \\
\text { en la asignatura fueron } \\
\text { aplicados en el producto final } \\
\text { entregado al socio comunitario. }\end{array}$ & $66,7 \%$ & $33,3 \%$ & $0 \%$ & $0 \%$ & $0 \%$ & $0 \%$ & $0 \%$ & $100 \%$ \\
\hline $\begin{array}{l}\text { La incorporación de la } \\
\text { metodología As en una } \\
\text { asignatura es una experiencia } \\
\text { recomendable para la docencia. }\end{array}$ & $66,7 \%$ & $33,3 \%$ & $0 \%$ & $0 \%$ & $0 \%$ & $0 \%$ & $0 \%$ & $100 \%$ \\
\hline $\begin{array}{l}\text { La incorporación de la } \\
\text { metodología As en una } \\
\text { asignatura es una experiencia } \\
\text { recomendable para el } \\
\text { estudiantado. }\end{array}$ & $66,7 \%$ & $33,3 \%$ & $0 \%$ & $0 \%$ & $0 \%$ & $0 \%$ & $0 \%$ & $100 \%$ \\
\hline $\begin{array}{l}\text { Su grado de satisfacción } \\
\text { general con la asignatura } \\
\text { implementada con } \\
\text { metodología As* }\end{array}$ & $41,7 \%$ & $41,7 \%$ & $8,3 \%$ & $8,3 \%$ & $0 \%$ & $0 \%$ & $0 \%$ & $100 \%$ \\
\hline
\end{tabular}

Nota: $7=$ Totalmente de acuerdo; 6 = De acuerdo; 5 = Moderadamente de acuerdo; 4 = Ni de acuerdo ni en desacuerdo; 3 = Moderadamente en desacuerdo; 2 = En desacuerdo; 1 = Totalmente en desacuerdo. En el caso del ítem señalados con $\left({ }^{*}\right): 7=$ Muy satisfecho; 6 = Satisfecho; $5=$ Moderadamente satisfecho; $4=$ Ni satisfecho ni insatisfecho;

3 = Moderadamente insatisfecho; 2 = Insatisfecho; $1=$ Muy insatisfecho.

Fuente: elaboración propia a partir de los datos del Proyecto Aprendizaje Servicio de la FACE.

Finalmente, un indicador del significado y el valor que los estudiantes le dan a este tipo de iniciativas se manifiesta en las estrategias de reflexión que se realizan tanto al inicio como al cierre de cada actividad. La reflexión inicial tiene por objetivo explorar las fortalezas y debilidades del estudiantado para desarrollar el servicio requerido; allí, se evidencia que las principales fortalezas identificadas por ellos a la hora de enfrentar este tipo de actividades son: la empatía, la responsabilidad, la paciencia y la perseverancia, mientras que las principales debilidades percibidas son: la impuntualidad, la inseguridad, la timidez y la impaciencia.

La estrategia de reflexión realizada al finalizar el servicio en cada asignatura se adecúa al número de experiencias de As que los mismos estudiantes han desarrollado a lo largo de su carrera, y busca profundizar en los aprendizajes experimentados al término del trabajo realizado en terreno, dando a conocer entre otras cosas lo aprendido a nivel tanto individual como grupal, y lo que se puede mejorar de la actividad desarrollada. En general, en esta instancia el estudiantado descubre la importancia 
de la empatía, la paciencia, la solidaridad, el trabajo en equipo, la conexión con otros y el conocimiento de otras realidades, entre otros aprendizajes, y reconoce entre las principales dificultades y aspectos a mejorar los problemas de comunicación, coordinación y organización que en ocasiones existen tanto dentro del equipo como con los beneficiarios, incluso, en algunos casos, con el mismo equipo coordinador.

Una de las reflexiones que identifica este proyecto es mencionada por un grupo de estudiantes y refleja la importancia de la institucionalización de esta metodología:

Aprendimos a buscar cómo ayudar y explicar de manera sencilla a una persona con menor conocimiento, pero con suficientes ganas de aprender y superarse [...] A través de la perseverancia de la emprendedora, cada integrante del equipo aprendió lo importante que es luchar por sus metas [...] Aprendimos que, a pesar de las dificultades, lo importante es perseverar. (Reflexión estudiantes de la carrera de Contador Público y Auditor)

Finalmente, el trabajo desarrollado en torno al proyecto Рм Uвв 1402 destaca la relevancia de la generación de un modelo de trabajo colaborativo con la comunidad, que contribuya al desarrollo de competencias genéricas y específicas del estudiantado y, a la vez, permita la consolidación de las redes de colaboración con organizaciones e instituciones públicas o privadas (Salazar et ál., 2019).

\section{Conclusiones}

A partir de las experiencias realizadas en la FACE, se puede afirmar que la incorporación de la metodología As en el aula brinda una oportunidad para avanzar en el desarrollo de competencias técnicas, valores y actitudes del estudiantado, para lograr un mayor compromiso social con el desarrollo territorial. Si bien esto se ha evidenciado en la teoría, es necesario constatar su aplicación en la práctica y observar cómo efectivamente la experiencia, la vivencia y la participación del estudiantado hacen una diferencia enorme en los aprendizajes técnicos y personales logrados.

Un factor de éxito para la implementación e institucionalización de esta metodología, evidenciado en esta experiencia, que beneficia al estudiantado, a los socios comunitarios y a los académicos, radica en el proceso de investigación-acción que implica la ejecución de las actividades. A pesar de que no es fácil visualizar los resultados en primera instancia, a medida que se avanza en el trabajo de implementación en el día a día se puede entender la complejidad que implica coordinar el trabajo de 
los estudiantes, los profesores y los beneficiarios. Al inicio se cometen muchos errores que poco a poco se interiorizan como aprendizajes permanentes, los cuales sin duda fortalecen la experiencia de quienes guían este trabajo. En el transcurso de este trabajo se aprecia lo imprescindible de la retroalimentación y de los ajustes a un primer periodo de implementación, con el objetivo de no desperdiciar la curva de aprendizaje construida. Los equipos de trabajo deben estar atentos a esta parte del proceso, porque de lo contrario es fácil cometer los mismos errores en una segunda implementación.

El proceso es algo que no hay que perder de vista en ningún momento. Se deben convocar constantemente reuniones de retroalimentación con espacios de consulta abiertos para todos los actores, la presencia en las asignaturas, el acompañamiento a docentes y la preparación del estudiantado, además de la atención a los requerimientos de socios beneficiarios. Para el equipo institucional este proceso significó un aprendizaje en la marcha, y tras un año de implementación se observaron dificultades por el solo hecho de descuidar temas de esta naturaleza. Se implementaron 29 cursos que contaron con la participación de 19 docentes, 276 socios y 901 estudiantes. En este sentido, es posible afirmar que se cumplió con el objetivo, aunque el éxito de la implementación de esta metodología no radica en la cantidad sino en la calidad, pues esto requiere tiempo, dedicación y compromiso.

En este proceso, es clave el papel del docente, pues es quien se lleva la mayor cantidad de trabajo; por esta razón, es muy importante que su participación sea voluntaria, con convicción y compromiso con esta metodología, pues las horas que deberá destinar para su desarrollo y la forma de trabajo con el estudiantado será totalmente diferente a la convencional. En consecuencia, es vital la institucionalización de la metodología; en tanto esta exista, se pueden ofrecer incentivos como la incorporación de horas en las cargas de trabajo y el apoyo de ayudantes, además de establecer procedimientos y políticas institucionales que respalden la implementación. Se ha comprobado que, si esta actividad no es incorporada en la carga académica de los docentes como parte de sus labores normales, con la asignación de horas directas e indirectas, el apoyo de asistentes para la asignatura y las condiciones administrativas necesarias, se complejiza el éxito de la implementación. Es imprescindible destacar que todo este esfuerzo implica un gran trabajo y dedicación, así como un costo de tiempo personal alto.

A nivel institucional, se debe contar con docentes motivados, interesados y con las habilidades para ejecutar los pasos críticos necesarios. Es importante que la institución realice un diagnóstico respecto al estado en el que se encuentra, su 
proyección, los recursos, los valores y la cultura que quiere impulsar. Además, debe asumir el liderazgo y la responsabilidad administrativa con la creación de una unidad de As que comunique y expanda estos proyectos hacia cada dependencia de la institución. Este es un desafío para futuras investigaciones o acciones.

La experiencia indica que debe existir un equipo de apoyo que respalde al profesor, al estudiantado y a los socios. El profesor debe concentrar sus fuerzas en el desarrollo de los contenidos de los cursos y su vinculación con las necesidades descritas, pero no gastar energías en temas administrativos, de procedimientos o de otra naturaleza que lo distraigan de lo central.

No es posible dejar solo al estudiantado en este proceso; es imperativo acompañarlos, orientarlos y ayudarlos a construir sus equipos de trabajo. También, es fundamental el apoyo para que se conecten socialmente entre ellos y con sus socios beneficiarios de manera habilidosa. Por lo anterior, es perentorio contar con el tiempo y con los equipos de trabajo para realizar esta labor. Uno de los temas más complejos de trabajar es el de comprender la magnitud del compromiso que se adquiere con un agente externo a la universidad, que espera el desarrollo de un producto que brinde una solución a un problema concreto en la realidad, no solo como un ejercicio más dentro de la carrera. Detrás de este esfuerzo hay socios beneficiarios con altas expectativas, porque esto puede repercutir en mejores resultados para su negocio.

La implementación de talleres de fortalecimiento de habilidades es uno de los resultados más destacados y acertados del proyecto. Por esta razón, se hizo un diagnóstico de las habilidades sociales del estudiantado (Salazar-Botello, Mendoza-Llanos y Muñoz-Jara, 2020), que se transformó en un insumo importante para tomar decisiones y definir estrategias de acción requeridas para implementar la metodología. Si bien los resultados no fueron exitosos en todos los casos, si se pudieron observar cambios en las formas de interacción y la disposición mutua del estudiantado, así como en sus relaciones con los docentes y los agentes externos.

Esta metodología carece de todo sentido si el resultado final es un producto que no responde a las necesidades originales de los socios beneficiarios. En este sentido, se debe procurar que los informes y presentaciones finales sean de la calidad que el proyecto amerita. Esta tarea no siempre es fácil, y puede convertirse en un problema cuando no se cumple, pues requiere de un gran compromiso por parte del estudiantado y de los profesores de las asignaturas que, en algunos casos, están condicionados por el tiempo, para cumplir con el programa de la asignatura y además no tienen el equipo de apoyo suficiente para el desarrollo de este trabajo. 
Lo anterior está en directa relación con la importancia que se le da al cumplimiento del programa de la asignatura, ya que con ello se da cuenta del aprendizaje y de la formalización de la metodología. En consecuencia, el compromiso, la calidad, el detalle, la responsabilidad, la dedicación, el respeto y la generosidad son cualidades importantes para este proceso. Por esta razón, una de las recomendaciones importantes para los estudiantes es que no se involucren semestralmente en más de una asignatura con esta metodología.

Todo esto muestra el alto nivel de detalle logístico que se requiere: el tiempo que se necesita para consolidar las actividades, el acompañamiento al estudiantado en el trabajo de campo y a los socios beneficiarios, así como el apoyo a los académicos y la sistematización de la información, para generar el modelo de institucionalización de la metodología As, sin olvidar la gestión administrativa para convencer a los tomadores de decisión, que es una tarea fundamental.

La finalidad de este trabajo en el mediano plazo consiste en mostrar lo relevante de la institucionalización de la metodología As en la Universidad del Bío-Bío, estableciendo equipos de trabajo multidisciplinario que acompañan a docentes, estudiantes y organizaciones comunitarias en el desarrollo de las iniciativas de trabajo colaborativo, impulsadas en el marco de este programa. La institucionalización significa aquí tomar decisiones de política universitaria y generar con ello los procedimientos y el apoyo estructural y real para su implementación. Este trabajo deja finalmente la puerta abierta para visualizar el impacto de esta metodología en el estudiantado y en la comunidad, así como en las formas de impartir docencia.

\section{Sobre los autores}

Carlos Mauricio Salazar-Botello. Magíster en Desarrollo y Comportamiento Organizacional por la Universidad Diego Portales, Chile; licenciado en Educación; ingeniero de ejecución en Administración de Empresas por la Universidad del Bío-Bío, Chile. Profesor asociado del Departamento de Gestión Empresarial y director del Plan de Mejoramiento UBB 1402 Aprendizaje Servicio de la Facultad de Ciencias Empresariales de la Universidad del Bío-Bío. Áreas de interés: gestión del cambio, cultura organizacional, gestión por competencias y habilidades sociales. Publicaciones recientes: "Impacto diferenciado del tiempo de formación universitaria según institución de educación media en el desarrollo de habilidades sociales (2020)"; "Necesidades de capacitación en una municipalidad de la zona central de Chile: la mirada de los funcionarios (2020)"; “Competencias genéricas en la Educación Superior: una experiencia en la Universidad del Bío-Bío, Chile (2019)”; “Contribuyendo a la visibilización de la metodología Aprendizaje Servicio en la Universidad del Bío-Bío: oportunidades y 
desafíos (2019)”; “Creencias organizacionales, satisfacción laboral y síndrome burnout en docentes de Educación Superior (2018)”.

Yohana Andrea Muñoz Jara. Magíster en Gestión de Empresas, ingeniera Comercial y licenciada en Ciencias Administrativas por la Universidad del Bío-Bío, Chile. Actualmente, se desempeña como coordinadora del Proyecto Aprendizaje Servicio de la Facultad de Ciencias Empresariales de la Universidad del Bío-Bío. Áreas de interés: aprendizaje, servicio y comportamiento organizacional. Publicaciones recientes: "Impacto diferenciado del tiempo de formación universitaria según institución de educación media en el desarrollo de habilidades sociales (2020)"; "Necesidades de capacitación en una municipalidad de la zona central de Chile: la mirada de los funcionarios (2020)"; "Competencias genéricas en la Educación Superior: una experiencia en la Universidad del Bío-Bío, Chile (2019)”; “Contribuyendo a la visibilización de la metodología Aprendizaje Servicio en la Universidad del Bío-Bío: oportunidades y desafíos (2019)”; “Creencias organizacionales, satisfacción laboral y síndrome burnout en docentes de Educación Superior (2018)”.

María Teresa Lagos Troncoso. Magíster en Gestión Educacional (IEDE y Universidad San Sebastián), Chile. Contador auditor por la Universidad del Bío-Bío y profesora de Estado en física y ciencias naturales de la misma universidad. Actualmente, se desempeña como profesional de apoyo del Proceso de Seguimiento y Acompañamiento de la Carrera de Contador Público y Auditor y coordinadora de la metodología aprendizaje servicio, sede Chillán, del Proyecto Aprendizaje Servicio de la Facultad de Ciencias Empresariales de la Universidad del Bío-Bío. Además, es docente de medio tiempo del Departamento de Gestión Empresarial. Áreas de interés: aprendizaje servicio y contabilidad. Publicaciones recientes: "Contribuyendo a la visibilización de la metodología Aprendizaje Servicio en la Universidad del Bío-Bío: oportunidades y desafíos (2019)”.

Raúl Luciano Arriagada Inostroza. Magíster en Educación, con mención en Gestión Curricular, por la Universidad del Bío-Bío, Chile. Profesor de matemática por la Universidad de Concepción. Actualmente, se desempeña como asesor metodológico de la Unidad de Formación Integral de la Universidad del Bío-Bío, sede Chillán, y como coordinador de la metodología aprendizaje servicio, sede Concepción, del Proyecto Aprendizaje Servicio de la Facultad de Ciencias Empresariales de la Universidad del Bío-Bío. Áreas de interés: educación. Publicaciones recientes: "La certificación de colegios: ¿un referente de la calidad? (2014)”; "Modelo de Guía Didáctica de Aprendizaje Autónomo Digital compatible con equipos móviles para la Universidad del Bío-Bío (2019)”.

Rosana Lorena Vallejos Cartes. Licenciada en Educación, con mención en Historia y Geografía, por la Universidad de Concepción, Chile; Master of Science Rural Planning and Development por la Universidad de Guelph, Canadá. Miembro de Wocan, Women Organization for Change in Agriculture y Natural Resource Management, así como de la Red de Aprendizaje Servicio Rease de Chile. Actualmente, trabaja en el Centro de Investigación en Economía y Desarrollo Agroalimentarios (Creda-IRTA-UPC) en su Doctorado en Sostenibilidad (Universidad Politécnica de Cataluña, España). Beneficiaria de la Beca INIA 2017-0028 (España) y Graduate Student Research Assistantships (Canadá, 2005). Áreas de interés: planificación y desarrollo rural; sistemas agroalimentarios y estudios del consumidor; mujer rural, emprendimiento y extensión rural; y Aprendizaje Servicio. Publicaciones recientes: "Exploring 
the linkage between secondary technical and vocational education system, labor market and family setting. A prospective analysis from central Chile (2020)"; "Presencia de las mujeres en la alta gestión universitaria. Las universidades públicas en Chile (2018)”.

Rodrigo Javier Monje -Sanhueza. Magíster en Dirección de Empresas, ingeniero comercial y licenciado en Ciencias Administrativas por la Universidad del Bío-Bío, Chile. Actualmente, se desempeña como coordinador de la Unidad de Gestión de Programas de la Dirección de Posgrado de la Universidad del Bío-Bío. Áreas de interés: dirección estratégica, control de gestión e investigación en docencia universitaria. Ha sido reconocido con el Premio Universidad del Bío-Bío que acredita excelencia en el rendimiento académico.

\section{Referencias}

Bringle, R. G. y Hatcher, J. A. (1996). Implementing service learning in higher education. Journal of Higher Education, 67(2), 221-239. https://doi.org/10.2307/2943981

Chaverri, P. (2015). El Aprendizaje en Servicio en Ulacit: conceptos, experiencias y retos. Universidad Latinoamericana de Ciencia y Tecnología.

Cooper, S. B., Cripps, J. H. y Reisman, J. I. (2013). Service-learning in deaf studies: Impact on the development of altruistic behaviors and social justice concern. American Annals of the Deaf, 157(5), 413-427. https://doi.org/10.1353/aad.2013.0003

Daura, F. (2011). Las estrategias docentes al servicio del desarrollo del aprendizaje autorregulado. Estudios Pedagógicos, 37(2), 77-88.

Dewey, J. (1938). Experiencie and education. Macmillan.

Escofet, A., Folgueiras, P., Luna, E. y Palou, B. (2016). Elaboration and Validation of a Questionnaire for the Evaluation of Service-Learning Projects. Revista Mexicana de Investigacion Educativa, 21(70), 929-949.

Eyler, J. (2009). The Power of Experiential Education. Liberal Education, 95(4), 24-31.

Ferrán-Zubillaga, A. y Guinot-Viciano, C. (2012). Aprendizaje-servicio: propuesta metodológica para trabajar competencias. Portularia, 12, 187-195. https://doi.org/10.5218/ prts.2012.0020

Francisco, A. y Moliner, L. (2010). El Aprendizaje Servicio en la Universidad: una estrategia en la formación de ciudadanía crítica. Revista Electrónica Interuniversitaria de Formación del Profesorado, 13(4), 69-77.

Freire, P. (2002). Pedagogía de la Esperanza. Un reencuentro con la pedagogía del oprimido. Siglo XXI Editores.

Furco, A. (2002). Self-Assessment Rubric for the Institutionalization of Service-Learning in Higher Education. Campus Compact. University of California. 
Furco, A. y Billig, S. (2002). Service-learning: The Essence of the Pedagogy. Information Age Publishing.

Godoy-Pozo, J., Illesca-Pretty, M., Seguel-Palma, F. y Salas-Quijada, C. (2019). Desarrollo y fortalecimiento de competencias genéricas en estudiantes de enfermería a través de la metodología aprendizaje-servicio. Rev. Fac. Med., 67(3), 449-458. https://doi.org/10.15446/ revfacmed.v67n3.69014

Groh, C. J., Stallwood, L. G., y Daniels, J. J. (2011). Service-learning in nursing education: Its impact on leadership and social justice. Nursing Education Perspectives, 32(6), 400-405. https://doi.org/10.5480/1536-5026-32.6.400

Hernández, R., Fernández, C. y Baptista, M. (2010). Metodología de la investigación (5. edición). McGraw-Hilll.

Jacoby, B. (1996). Service-learning in higher education: concepts and practices. Jossey-Bass Publishers.

Jenkins, A. y Sheehey, P. (2011). A Checklist for Implementing Service-Learning in Higher Education. Journal of Community Engagement and Scholarship, 4(2), 52-60.

Jouannet, C., Montalva, J., Ponce, C. y Von Borries, V. (2015). Diseño de un modelo de institucionalización de la metodología de aprendizaje servicio en educación superior. Revista Iberoamericana de Aprendizaje Servicio (Ridas), (1), 112-131. https://doi.org/10.1344/ RIDAS2015.1.7

Jouannet, C., Salas, M. y Contreras, M. (2013). Modelo de implementación de Aprendizaje Servicio (A+S) en la UC. Una experiencia que impacta positivamente en la formación profesional integral. Calidad en la Educación, (39), 198-212.

Martínez, M. (Ed.). (2010). Aprendizaje servicio y responsabilidad social de las universidades. Octaedro.

Martínez-Odria, A. (2007). Service-learning o aprendizaje-servicio. La apertura de la escuela a la comunidad local como propuesta de educación para la ciudadanía. Bordón. Revista de Pedagogía, 59(4), 627-640.

Mayor, D. (2013). Aprender realizando una actividad de utilidad social. Guía básica para el diseño de proyectos de Aprendizaje-Servicio. Revista de Educación Social, (16), 1-14.

Mayor, D. (2018). Aprendizaje-Servicio: una práctica educativa innovadora que promueve el desarrollo de competencias del estudiantado universitario. Revista Electrónica Actualidades Investigativas En Educación, 18(3), 1-22. https://doi.org/10.15517/aie.v18i3.34418

Mayor, D. (2019). Dimensiones pedagógicas que configuran las prácticas de aprendizaje-servicio. Revista Páginas de Educación, 12(2), 23-42. https://doi.org/10.22235/pe.v12i2.1834

Mayor, D. y Rodríguez, D. (2015). Aprendizaje-Servicio: construyendo espacios de intersección entre la escuela-comunidad-universidad. Profesorado. Revista de Curriculum y Formación de Profesorado, 19(1), 262-279. 
Institucionalización del aprendizaje servicio en la Facultad de Ciencias Empresariales...

Mayor, D. y Rodríguez, D. (2017). Aprendizaje-Servicio: una práctica pedagógica que promueve la participación del estudiantado para la mejora escolar y social. Revista Complutense de Educación, 28(2), 555-571. https://doi.org/10.5209/rev_RCED.2017.v28.n2.49623

Mecesup. (2015). Financiamiento institucional: planes de mejoramiento de programas (PM). http://www.mecesup.cl/index2.php?id_seccion=5194yid_portal=59yid_contenido=31511

Ochoa, A. y Pérez, L. (2019). El aprendizaje servicio, una estrategia para impulsar la participación y mejorar la convivencia escolar. Psicoperspectivas, 18(1), 1-13. https://doi. org/10.5027/psicoperspectivas-vol18-issue1-fulltext-1478

Páez, M. y Puig, J. (2013). La reflexión en el Aprendizaje-Servicio. Revista Internacional de Educación Para La Justicia Social, 2(2), 13-32. http://www.rinace.net/riejs/numeros/vol2num2/art1.pdf

Pérez, L. y Ochoa, A. (2017). El aprendizaje-servicio (APS) como estrategia para educar en ciudadanía. Alteridad. Revista de Educación, 12(2), 175-187. https://doi.org/10.17163/alt. v12n2.2017.04

Pizarro, V., y Hasbún, B. (Eds.). (2019). Aprendizaje Servicio en la Educación Superior Chilena. https://doi.org/10.34720/WCC5-5184

Puig, J. (Ed.). (2009). Aprendizaje Servicio (ApS). Educación y compromiso cívico. Graó.

Puig, J., Batlle, R., Bosch, C. y Palos, J. (2007). Aprendizaje Servicio: educar para la ciudadanía. Octaedro.

Puig, J., Gijón, M., Martín, X. y Rubio, L. (2011). Aprendizaje-servicio y educación para la ciudadanía. Revista de Educación, (extraordinario), 45-67.

Puig, J. y Palos, J. (2006). Rasgos pedagógicos del Aprendizaje-Servicio. Cuadernos de Pedagogía, 357, 60-63.

Reimers, F. y Chung, C. (2016). Enseñanza y aprendizaje en el siglo XXI: Metas, políticas educativas y currículo en seis países. Fondo de Cultura Económica.

Rodríguez-Gallego, M. (2014). El Aprendizaje-Servicio como estrategia metodológica en la Universidad. Revista Complutense de Educación, 25(1), 95-113. https://doi.org/https://doi. org/10.5209/rev_RCED.2014.v25.n1.41157

Salazar-Botello, M., Mendoza-Llanos, R. y Muñoz-Jara, Y. (2020). Impacto diferenciado del tiempo de formación universitaria según institución de educación media en el desarrollo de habilidades sociales. Propósitos y Representaciones, 8(2). https://doi.org/http:// dx.doi.org/10.20511/pyr2020.v8n2.416

Salazar, C., Vallejos, R., Muñoz, Y.y Lagos, M. T. (2019). Contribuyendo a la visibilización de la metodología aprendizaje servicio en la Universidad del Bío-Bío: oportunidades y desafíos. En V. Pizarro y B. Hasbún (Eds.), Aprendizaje Servicio en la Educación Superior Chilena (1.a edición, pp. 194-205). Ediciones CEA-FEN Universidad de Chile.

Salazar, R. (2016). Los pilares para la educación superior del futuro: responsabilidad social, calidad académica y servicio-aprendizaje (S-A). Fides et Ratio, 11, 155-179. 
Sandrea, L. y Reyes, L. (2010). Proyectos de aprendizaje de servicio-comunitario y su influencia en las conductas prosociales de estudiantes universitarios. Revista de Pedagogía, 31(89), 379-401.

Stake, R. (2007). Investigación con estudio de casos. Ediciones Morata.

Tapia, M. (2001). La solidaridad como pedagogía (2. a edición). Ciudad Nueva.

Tapia, M. (2010). La propuesta pedagógica del “Aprendizaje-Servicio”: una perspectiva Latinoamericana. Revista Tzhoecoen, 3(5), 23-43.

Tapia, M. (2014). Aprendizaje y servicio solidario: en el sistema educativo y las organizaciones juveniles (1. ${ }^{\mathrm{a}}$ edición). Ciudad Nueva.

Tapia, M., Amar, H., Montes, R., Tapia, M. R. y Yaber, L. (2013). Manual para docentes y estudiantes solidarios ( $3 .^{a}$ edición). Clayss.

Tapia, M., Bridi, G., Maidana, M. y Rial, S. (2015). El compromiso social como pedagogía. Aprendizaje y solidaridad en la escuela. Celam.

Universidad del Bío-Bío. (2008). Modelo Educativo de la Universidad del Bío-Bío. http://www. ubiobio.cl/web/descargas/Modelo_Educativo_(08.07.08).pdf

Uribe, P. (2018). Percepción de los estudiantes de educación inicial frente al desarrollo de experiencias formativas en modalidad A+S. Revista Electrónica de Investigación Educativa, 2O(4), 110-122. https://doi.org/10.24320/redie.2018.20.4.1826

Uruñuela, P. (2011). Aprendizaje-Servicio, una metodología para el desarrollo de la convivencia. Revista AMAzônica, 6(1), 35-61.

Vallejos, R., Salazar, C., Muñoz, Y., Arriagada, R. y Roca, S. (2016). Aprendizaje servicio: emprendiendo e innovando en educación superior. La experiencia de la Universidad del

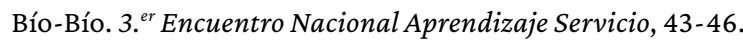

